\title{
Stability of a shallow arch with one end moving at constant speed
}

\author{
Jen-San Chen*, Jian-San Lin \\ Department of Mechanical Engineering, National Taiwan University, Taipei 10617, Taiwan
}

Received 25 March 2004; received in revised form 1 April 2006; accepted 6 April 2006

\begin{abstract}
In this paper we consider a shallow arch with rise parameter $h$, free of lateral loading, but subject to prescribed end motion $e$ with constant speed $c$. Attention is focused on finding out whether dynamic snap-through will occur. Quasi-static analysis is first performed to identify all equilibrium configurations and their stability properties when $e$ and $h$ are specified. If the arch is stretched quasi-statically, it will be straightened up and no snap-through will occur. However, when the speed $c$ is not negligible it is possible for the arch to snap to the other side dynamically. Careful analysis shows that the only possible situation when dynamic snap-through may occur is $h>3 \sqrt{6}$ and $\sqrt{\frac{8}{3}} h-4<e<\frac{2}{9} h^{2}-4$. In this case, to prevent dynamic snap-through to occur the end speed $c$ must not exceed a critical speed, which is a function of $e$ and $h$. The minimum critical stretching speed is found to be 25.9 for all possible combinations of $e$ and $h$.
\end{abstract}

(C) 2006 Elsevier Ltd. All rights reserved.

Keywords: Dynamic stability; Shallow arch; Energy barrier; End motion

\section{Introduction}

One important response of a shallow arch under transverse loading is snap-through buckling. The buckling phenomenon is characterized by a sudden jump from one equilibrium configuration to another for which the displacements are distinctly larger than the first one. The researches in the stability of shallow arches may be divided into two categories, i.e., static stability and dynamic stability. In the aspect of static stability, the lateral loading is assumed to be applied in a quasi-static manner. Due to the non-linearity inherent in arch deformation, multiple stable equilibrium positions may exist and arch configuration may jump from one stable equilibrium position to another stable position. Early classical investigation can be found in [1-9]. Experimental results have been reported by Roorda [10].

In the case when the lateral loads are applied suddenly instead of in quasi-static manner, the phenomenon is dynamic and much more complicated. More importantly, the critical loads predicted will be different from the one predicted statically. For instance, if a lateral load is applied suddenly, the critical load will be about $80 \%$ of the one applied quasi-statically. In

\footnotetext{
* Corresponding author. Tel.: +886223661734; fax: +886223631755.

E-mail address: jschen@ccms.ntu.edu.tw (J.-S. Chen).
}

other words, it is easier to snap the arch to the other side if the lateral load is applied suddenly instead of quasi-statically. A necessary and sufficient condition for dynamic stability is in general difficult to obtain because it requires a complete integration of the non-linear partial differential equation of motion. However, if we ignore the history of motion then we may find a sufficient stability condition against snap-through. This approach has been adopted in many dynamic stability researches of shallow arches, for instance, [11-28]. In all these analyses, both ends of the shallow arches are fixed in space.

In this paper, we investigate a new elastic stability problem which involves a shallow arch under prescribed end motion. The arch is assumed to be free of lateral loading. At time $t=0$ we assume that one end of the arch starts to move to a new position with constant speed, while the other end remains fixed in space. When the end speed is very small, the inertial effect and the kinetic energy of the arch can be neglected. In this case it is easy to visualize that the arch will be "straightened up", and it is unlikely that the arch will be snapped to the other side. However, when the end speed is not negligible and inertial effect has to be taken into account, we wish to find out whether it is possible for the arch to be snapped to the other side dynamically. This problem not only is new from the 
academic point of view, it may have practical application as well. Consider an arch structure designed and constructed to be in a stable equilibrium position. In the case when certain unexpected disturbance occurs such that the distance between the two ends of the arch increases, it is important to predict whether the structure still holds or not.

\section{Equations of motion}

Fig. 1 shows an elastic shallow arch with the two pinned ends separated originally by a distance $L$. The initial shape of the arch is $y_{0}(x)$. At time $t=0$, one of the ends starts to move a distance $\delta$ with constant speed $c^{*}$. The shape of the stretched arch is $y(x, t)$. It is noted that we prescribe only the end motion, and assume that the external force needed for this motion is always available. The equation of motion of the arch can be written as

$\rho A y_{, t t}=-E I\left(y-y_{0}\right)_{, x x x x}+p^{*} y_{, x x}$.

The parameters $E, \rho, A$, and $I$ are Young's modulus, mass density, area, and area moment of inertia of the cross section of the arch. $p^{*}$ is the axial force,

$p^{*}(t)=\frac{A E}{L}\left[\delta(t)+\frac{1}{2} \int_{0}^{L}\left(y_{, x}^{2}-y_{0, x}^{2}\right) \mathrm{d} x\right]$.

In writing Eqs. (1) and (2) we neglect the inertial effect in the axial direction. The boundary conditions for $y$ at $x=0$ and $L$ are

$$
\begin{aligned}
y(0)-y_{0}(0) & =y_{, x x}(0)-y_{0, x x}(0)=y(L)-y_{0}(L) \\
& =y_{, x x}(L)-y_{0, x x}(L)=0 .
\end{aligned}
$$

Eqs. (1) and (2) can be non-dimensionalized to the forms

$u_{, \tau \tau}=-\left(u-u_{0}\right)_{, \xi \xi \xi \xi}+p u_{, \xi \xi}$

$p=e+\frac{1}{2 \pi} \int_{0}^{\pi}\left(u_{, \xi}^{2}-u_{0, \xi}^{2}\right) \mathrm{d} \xi$

where

$$
\begin{aligned}
& u=\frac{y}{r}, \quad u_{0}=\frac{y_{0}}{r}, \quad \xi=\frac{\pi x}{L}, \quad \tau=\frac{\pi^{2} t}{L^{2}} \sqrt{\frac{E I}{A \rho}}, \\
& p=\frac{p^{*} L^{2}}{\pi^{2} E I}, \quad e=\frac{L \delta}{\pi^{2} r^{2}} .
\end{aligned}
$$

Here $r$ is the radius of gyration of the cross section. $p=1$ coresponds to the Euler buckling load for a perfectly straight simply supported beam. The initial shape of the arch is assumed to be in the form

$u_{0}=h \sin \xi$,

where $h$ is the rise parameter of the arch. With this initial shape, we can calculate the maximum stretching distance $e_{\max }$ if the beam is assumed to be inextensible,

$e_{\max }=\frac{h^{2}}{4}$

Eq. (7) corresponds to the case when the curved beam is stretched to a straight line. All the stretching distance $e$ discussed in this paper is limited to be smaller than $e_{\max }$.

It is assumed that the shape of the arch after stretching can be expanded

$u(\tau)=u_{0}+\sum_{n=1}^{\infty} \alpha_{n}(\tau) \sin n \xi$.

After substituting Eqs. (6) and (8) into (4) and (5) we obtain the equations governing $\alpha_{n}$,

$\ddot{\alpha}_{1}=-\alpha_{1}-(G+e)\left(h+\alpha_{1}\right)$,

$\ddot{\alpha}_{n}=-n^{4} \alpha_{n}-n^{2}(G+e) \alpha_{n}, \quad n=2,3, \ldots$,

where

$G=\frac{1}{4} \sum_{k=1}^{\infty} k^{2} \alpha_{k}^{2}+\frac{h}{2} \alpha_{1}$,

$e(\tau)=c \tau$.

The dimensionless speed $c$ is related to $c^{*}$ by

$c=\frac{L^{3}}{\pi^{4} r^{3}} \frac{c^{*}}{c_{l}}$

where $c_{l}$ is the longitudinal wave speed of the arch. The overhead dots in Eqs. (9) and (10) represent differentiation with respect to $\tau$. The axial force $p$ in Eq. (5) can be calculated as

$p=G+e$.

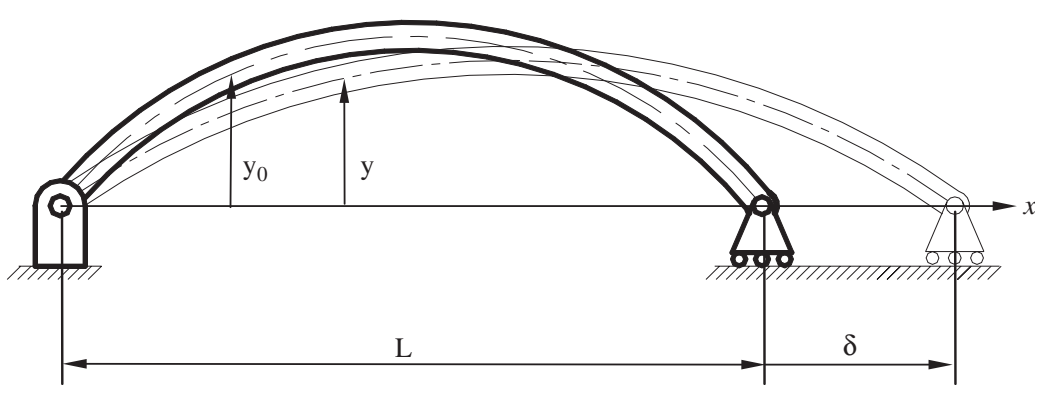

Fig. 1. Schematic diagram of a shallow arch under prescribed end motion. 


\section{Equilibrium configurations}

We first assume that the speed $c$ is small and the acceleration terms in Eqs. (9) and (10) can be neglected. From this condition we can examine the equilibrium configurations for various values of $e$. There are two different types of solutions, i.e., oneand two-mode solutions. The one-mode solution involves only the first mode $\alpha_{1} \sin \xi$, while the two-mode solution involves the first mode $\alpha_{1} \sin \xi$ and one of the higher modes $\alpha_{j} \sin j \xi$. The properties of these two types of solutions are discussed in detail in the following:

One-mode solution $u=u_{0}+\alpha_{1} \sin \xi$ :

In this case $\alpha_{1}$ satisfies the following cubic equation:

$f\left(\alpha_{1}\right)=g\left(\alpha_{1}\right)$,

where

$f\left(\alpha_{1}\right)=\frac{\alpha_{1}}{4}\left(\alpha_{1}^{2}+3 h \alpha_{1}+2 h^{2}+4\right)$

$g\left(\alpha_{1}\right)=-e\left(\alpha_{1}+h\right)$.

It is noted that $f\left(\alpha_{1}\right)$ is independent of $e$, while $g\left(\alpha_{1}\right)$ is linear in $e$. Fig. 2 shows several possible situations of the cubic curve $f\left(\alpha_{1}\right)$ and the straight line $g\left(\alpha_{1}\right)$. First of all, we note that $f\left(\alpha_{1}\right)$ always passes points $(0,0)$ and $(-h,-h)$. Furthermore, $f\left(\alpha_{1}\right)$ crosses the $\alpha_{1}$-axis at three distinct points only when $h>4$. Since $e$ is always positive in our discussion, the straight line $g\left(\alpha_{1}\right)$ always passes point $(-h, 0)$ with negative slope. From Eqs. (16) and (17) we observe that $g\left(\alpha_{1}\right)$ touches $f\left(\alpha_{1}\right)$ when $e=e_{1}$, where

$e_{1}=\frac{h^{2}}{4}-\frac{3}{4}(2 h)^{2 / 3}-1$.

It is noted that $e_{1}=0$ when $h=4$. Since the intersections of $f\left(\alpha_{1}\right)$ and $g\left(\alpha_{1}\right)$ represent the equilibrium configurations, we can make the following observations:

(1) If $h<4$, or $h \geqslant 4$ but $e>e_{1}$, then there is only one intersection in Fig. 2. This equilibrium configuration is denoted by $P_{0}$. Apparently,

$-h<\alpha_{1}\left(P_{0}\right)<0$.

(2) If $h>4$ and $e<e_{1}$, then there are three intersections in Fig. 2. The corresponding equilibrium configurations are denoted by $P_{0}, P_{1}^{+}$and $P_{1}^{-}$, respectively. The corresponding $\alpha_{1}$ for $P_{1}^{+}$and $P_{1}^{-}$are in the ranges

$-h-(2 h)^{1 / 3}<\alpha_{1}\left(P_{1}^{+}\right)<-\frac{3}{2} h+\frac{1}{2} \sqrt{h^{2}-16}$,

$-\frac{3}{2} h-\frac{1}{2} \sqrt{h^{2}-16}<\alpha_{1}\left(P_{1}^{-}\right)<-h-(2 h)^{1 / 3}$.

The locations of these three equilibrium configurations are shown in Fig. 2.

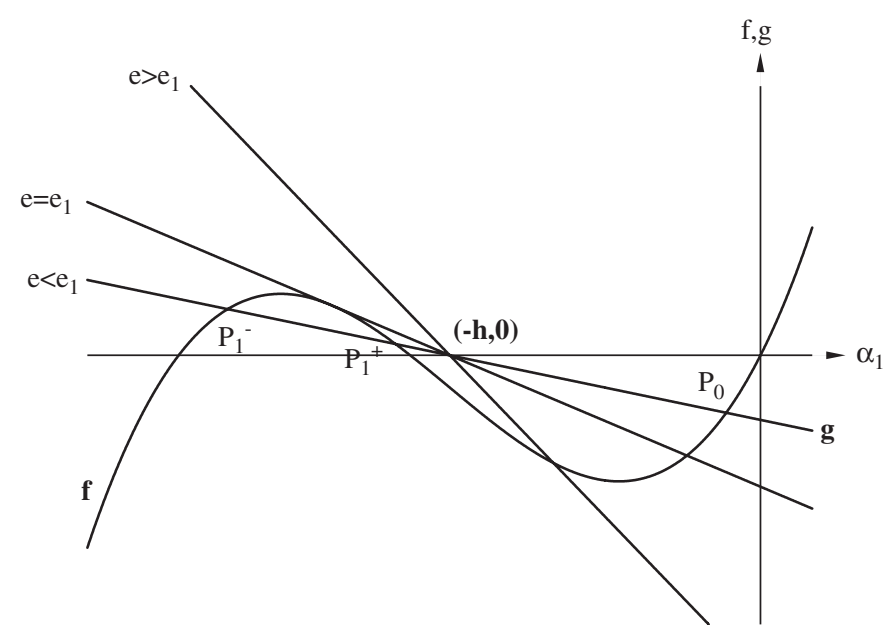

Fig. 2. $f\left(\alpha_{1}\right)$ and $g\left(\alpha_{1}\right)$ of the one-mode solution.

(3) If $e=e_{1}$, then the configurations $P_{1}^{+}$and $P_{1}^{-}$coincide. We denote this special one-mode configuration as $P_{1}$. Also,

$\alpha_{1}\left(P_{1}\right)=-h-(2 h)^{1 / 3}$.

Two-mode solution $u=u_{0}+\alpha_{1} \sin \xi+\alpha_{j} \sin j \xi$ :

For this case Eq. (9) becomes

$G+e=\frac{-\alpha_{1}}{\alpha_{1}+h}$.

In addition, the equation for $n=j$ in Eq. (10) becomes

$G+e=-j^{2}$.

By combining Eqs. (23) and (24) the solution $\alpha_{1}$ can be written explicitly as

$\alpha_{1}=\frac{-j^{2} h}{j^{2}-1}$.

After substituting Eq. (25) back into Eq. (24), the solution $\alpha_{j}$ can be solved as

$\alpha_{j}= \pm \frac{2}{j} \sqrt{e_{j}-e}$

where

$e_{j}=\frac{\left(j^{2}-2\right) j^{2} h^{2}}{4\left(j^{2}-1\right)^{2}}-j^{2}$.

These configurations are denoted by $P_{1 j}^{+}$and $P_{1 j}^{-}$, which exist only when

$e<e_{j}$

Since the $\alpha_{1}$ for $P_{1 j}^{+}$and $P_{1 j}^{-}$are the same, sometimes we write it as $\alpha_{1}\left(P_{1 j}^{ \pm}\right)$. It is noted that both the $e_{1}$ in Eq. (18) and $e_{j}$ in Eq. (27) are functions of $h$ only. By comparing Eqs. (18) and 
(27) we observe that

$e_{1} \geqslant e_{j}$

The special $h$ which renders $e_{1}=e_{j}$ is denoted by $\bar{h}_{j}$, where

$\bar{h}_{j}^{2}=2\left(j^{2}-1\right)^{3}, \quad j=2,3,4, \ldots$.

The values of some of $\bar{h}_{j}$ are $\bar{h}_{2}=3 \sqrt{6}, \bar{h}_{3}=32$, etc.

It is impossible for a solution to involve more than two higher modes. This can be proved by assuming that the solution involves $\alpha_{j} \sin j \xi$ and $\alpha_{k} \sin k \xi$, where both $j$ and $k$ are greater than 1 and $j \neq k$. After substituting the assumed solution into Eq. (9) for $n=j$ and $k$, one can prove that both equations can be satisfied simultaneously only when $j=k$. This clearly contradicts our original assumption. Therefore, it is impossible to have an equilibrium configuration involving more than two higher modes.

\section{Stability properties}

First of all, the dimensionless total energy $H$ of any configuration can be calculated as

$H=\frac{2}{\pi} \int_{0}^{\pi}\left[\left(u_{, \tau}\right)^{2}+\left(u, \xi \xi-u_{0, \xi \xi}\right)^{2}\right] \mathrm{d} \xi+2 p^{2}$.

The two terms in the integral represent the kinetic energy and the bending strain energy. The last term on the right-hand side is the strain energy due to the axial force. For an equilibrium configuration, the kinetic energy is zero and the total energy consists of only the strain energy $U$. The physical total energy $H^{*}$ and strain energy $U^{*}$ are related to $H$ and $U$ by

$H^{*}=\frac{\pi^{4} E I^{2} H}{4 A L^{3}}, \quad U^{*}=\frac{\pi^{4} E I^{2} U}{4 A L^{3}}$.

After substituting Eqs. (6), (8), and (14) into (31), $H$ and $U$ can be calculated as

$H=2(G+e)^{2}+\sum_{n=1}^{\infty}\left[\dot{\alpha}_{n}^{2}+n^{4} \alpha_{n}^{2}\right]$

$U=2(G+e)^{2}+\sum_{n=1}^{\infty} n^{4} \alpha_{n}^{2}$

For the two-mode configurations $P_{1 j}^{+}$and $P_{1 j}^{-}$the strain energy are equal, and can be written in terms of $e$ and $h$ as

$U\left(P_{1 j}^{+}\right)=U\left(P_{1 j}^{-}\right)=\frac{j^{4} h^{2}}{j^{2}-1}-2 j^{4}-4 j^{2} e$.

In order to study the stability of the equilibrium configuration with shape $\bar{u}$, we perturb the equilibrium shape by a small amount $\varepsilon \hat{u}$ to examine how the strain energy changes. $\varepsilon$ is a small positive number. The strain energy $U$ of the perturbed configuration can be expanded in terms of $\varepsilon$ as

$$
\begin{aligned}
U(\bar{u}+\varepsilon \hat{u})-U(\bar{u}) \\
=\varepsilon\left\{\frac{4}{\pi} \int_{0}^{\pi}\left[\left(\bar{u}_{, \xi \xi}-u_{0, \xi \xi)} \hat{u}_{, \xi \xi}+\bar{p}_{, \xi}, \hat{u}_{, \xi}\right] \mathrm{d} \xi\right\}\right. \\
+\varepsilon^{2}\left\{\frac{2}{\pi} \int_{0}^{\pi}\left[\left(\hat{u}_{, \xi \xi}\right)^{2}+\bar{p}\left(\hat{u}_{, \xi}\right)^{2}\right] \mathrm{d} \xi\right. \\
\left.+\frac{2}{\pi^{2}}\left[\int_{0}^{\pi} \bar{u}_{, \xi} \hat{u}_{, \xi} \mathrm{d} \xi\right]^{2}\right\} \\
+\varepsilon^{3}\left\{\frac{2}{\pi^{2}}\left[\int_{0}^{\pi} \bar{u}_{, \xi} \hat{u}, \xi \mathrm{d} \xi\right]\left[\int_{0}^{\pi}\left(\hat{u}_{, \xi}\right)^{2} \mathrm{~d} \xi\right]\right\} \\
+\varepsilon^{4}\left\{\frac{1}{2 \pi^{2}}\left[\int_{0}^{\pi}\left(\hat{u}_{, \xi}\right)^{2} \mathrm{~d} \xi\right]^{2}\right\} .
\end{aligned}
$$

$\bar{p}$ is the axial force of the equilibrium configuration, and can be calculated from Eqs. (9) and (14) as

$\bar{p}=\frac{-\alpha_{1}}{\alpha_{1}+h}$.

To prove that an equilibrium shape $\bar{u}$ is stable we have to show that the energy difference $U(\bar{u}+\varepsilon \hat{u})-U(\bar{u})$ is positive for any $\hat{u} \neq 0$. On the other hand, to prove that $\bar{u}$ is an unstable equilibrium shape, we only need to find one $\hat{u} \neq 0$ which renders $U(\bar{u}+\varepsilon \hat{u})-U(\bar{u})$ negative. After integrating by parts and using the fact that $\bar{u}$ satisfies the static equilibrium equation it can be shown that the coefficient of $\varepsilon$ in Eq. (36) is zero. To determine the stability we next examine the second variation of the strain energy

$\delta^{2} U=\frac{2}{\pi} \int_{0}^{\pi}\left[\left(\hat{u}_{, \xi \xi}\right)^{2}+\bar{p}\left(\hat{u}_{, \xi}\right)^{2}\right] \mathrm{d} \xi+\frac{2}{\pi^{2}}\left[\int_{0}^{\pi} \bar{u}_{, \xi} \hat{u}_{, \xi} \mathrm{d} \xi\right]^{2}$.

In some cases $\delta^{2} U$ is zero identically, and higher order variation is needed.

Theorem 4.1. Equilibrium configuration $P_{0}$ is stable.

Proof. Since $-h<\alpha_{1}\left(P_{0}\right)<0$ from Eq. (19), the axial force $\bar{p}$ in Eq. (37) is always positive. As a consequence $\delta^{2} U$ of $P_{0}$ is positive definite in $\hat{u}$, and $P_{0}$ is stable.

Theorem 4.2. Equilibrium configuration $P_{1}^{+}$is unstable.

Proof. For this case we consider $\hat{u}$ of the form

$\hat{u}=a_{1} \sin \xi$.

The second variation $\delta^{2} U$ can then be rewritten as

$\delta^{2} U=\frac{\left(\alpha_{1}+h\right)^{3}+2 h}{2\left(\alpha_{1}+h\right)} a_{1}^{2}$.

From Eq. (20) we can show that

$\frac{\left(\alpha_{1}+h\right)^{3}+2 h}{2\left(\alpha_{1}+h\right)}<0$ 
in the case when $e<e_{1}$. Therefore, $P_{1}^{+}$is unstable when $e<e_{1}$. In the case when $e=e_{1} \delta^{2} U$ is identically zero with respect to $a_{1}$. We proceed to calculate $\delta^{3} U$ as

$\delta^{3} U=\frac{1}{2}\left(\alpha_{1}+h\right) a_{1}^{3}$.

$\delta^{3} U$ is not zero except for $a_{1}=0$. Therefore, $P_{1}^{+}$is also unstable when $e=e_{1}$.

Theorem 4.3. Equilibrium configuration $P_{1}^{-}$is stable if and only if $e<e_{1}$ and $\bar{p}+4>0$.

Proof. This statement of necessary and sufficient condition is equivalent to the following four statements: (i) If $e=e_{1}$ then $P_{1}^{-}$is unstable. (ii) If $e<e_{1}$ and $\bar{p}+4>0$ then $P_{1}^{-}$is stable. (iii) If $e<e_{1}$ and $\bar{p}+4<0$ then $P_{1}^{-}$is unstable. (iv) If $e<e_{1}$ and $\bar{p}+4=0$ then $P_{1}^{-}$is unstable.

For the case $e=e_{1}$, configurations $P_{1}^{-}$and $P_{1}^{+}$are identical, and we have proven in Theorem 4.2 that $P_{1}^{+}$is always unstable. Therefore, statement (i) is proved. We next expand $\hat{u}$ in the form

$\hat{u}=\sum_{n=1}^{\infty} a_{n} \sin n \xi$

The second variation $\delta^{2} U$ can then be written as

$\delta^{2} U=\frac{\left(\alpha_{1}+h\right)^{3}+2 h}{2\left(\alpha_{1}+h\right)} a_{1}^{2}+\sum_{n=2}^{\infty}\left(n^{2}+\bar{p}\right) n^{2} a_{n}^{2}$.

For the case $e<e_{1}$ the terms involving $a_{1}^{2}$ is always positive due to Eq. (21). If $\bar{p}+4>0$, then every term in the summation involving $a_{n}^{2}$ is always positive. Therefore, $\delta^{2} U$ is positive definite and statement (ii) is proved. For the case $\bar{p}+4<0$, the coefficient of $a_{2}^{2}$ in Eq. (43) is negative. Therefore, $\delta^{2} U$ is not positive definite and statement (iii) is proved. For the last case $\bar{p}+4=0$ we consider $\hat{u}$ in the form

$\hat{u}=a_{1} \sin \xi+a_{2} \sin 2 \xi$.

By specifying $a_{2}=\eta \sqrt{a_{1}}$, one can rewrite the energy difference as

$$
\begin{aligned}
U(\bar{u}+\hat{u})-U(\bar{u})= & \left(\frac{h^{2}-54}{18}-\frac{2 h}{3} \eta^{2}+2 \eta^{4}\right) a_{1}^{2} \\
& + \text { high order terms. }
\end{aligned}
$$

Since it is always possible to find some $\eta$ to make the coefficient of $a_{1}^{2}$ negative, statement (iv) is proved.

Theorem 4.4. If $h \leqslant 3 \sqrt{6}$, then $P_{1}^{-}$is stable if and only if $e<e_{1}$. On the other hand, if $h>3 \sqrt{6}$, then $P_{1}^{-}$is stable if and only if $e<e_{2}$.

Proof. We first rewrite Eq. (37) into the following form:

$\bar{p}+4=\frac{-3}{\alpha_{1}+h}\left[-\alpha_{1}-\frac{4}{3} h\right]$.
It is noted that the factor $-3 /\left(\alpha_{1}+h\right)$ is always positive for $P_{1}^{-}$. The term $-\frac{4}{3} h$ in the bracket is equal to $\alpha_{1}\left(P_{12}^{ \pm}\right)$from Eq. (25). In the case when $h \leqslant 3 \sqrt{6}$, we can prove from Eqs. (22) and (25) that

$-h-(2 h)^{1 / 3} \leqslant-\frac{4 h}{3}$.

From Eqs. (21) and (47) we have $-\alpha_{1}\left(P_{1}^{-}\right)-\frac{4}{3} h>0$. Therefore, $\bar{p}+4>0$ and $P_{1}^{-}$is stable. In the other case when $h>3 \sqrt{6}$, following similar argument we have

$\alpha_{1}\left(P_{1}^{-}\right) \lesseqgtr \alpha_{1}\left(P_{12}^{ \pm}\right)=-\frac{4 h}{3}$ if and only if $e \lesseqgtr e_{2}$.

From Eqs. (46) and (48), and referring back to Theorem 4.3, the proof of Theorem 4.4 is obvious.

Theorem 4.5. Equilibrium configurations $P_{1 j}^{+}$and $P_{1 j}^{-}$are unstable.

Proof. For this case we consider the $\hat{u}$ in the form

$\hat{u}=a_{1} \sin \xi+a_{j} \sin j \xi$.

The second variation $\delta^{2} U$ can then be rewritten as

$\delta^{2} U=\left(1-j^{2}\right) a_{1}^{2}+\frac{1}{2}\left[\left(\alpha_{1}+h\right) a_{1}+j^{2} \alpha_{j} a_{j}\right]^{2}$.

Eq. (50) can also be expressed in the form

$\delta^{2} U=\mathbf{a M a}^{\mathrm{T}}$,

where the vector $\mathbf{a}$ and matrix $\mathbf{M}$ are defined as

$\mathbf{a}=\left(a_{1}, a_{j}\right)$,

$\mathbf{M}=\left(\begin{array}{ll}\frac{h^{2}-\bar{h}_{j}^{2}}{2\left(j^{2}-1\right)^{2}} & \frac{-h j^{2} \alpha_{j}}{2\left(j^{2}-1\right)} \\ \frac{-h j^{2} \alpha_{j}}{2\left(j^{2}-1\right)} & \frac{j^{4} \alpha_{j}^{2}}{2}\end{array}\right)$.

Since

$\operatorname{det} \mathbf{M}=-\frac{j^{4} \alpha_{j}^{2} \bar{h}_{j}^{2}}{4\left(j^{2}-1\right)^{2}}<0$,

$\delta^{2} U$ can be negative for certain non-trivial a. Therefore, $P_{1 j}^{+}$ and $P_{1 j}^{-}$are unstable.

The above five theorems completely establish the stability properties of all possible equilibrium configurations of a shallow arch under specified end movement $e$. Fig. 3 shows the bifurcation set in the $h-e$ plane. These $e_{j}$-curves divide the $h-e$ plane into several regions. For a given set of $h$ and $e$, we can determine how many equilibrium configurations exist in each region by the rules described in Section 3. For instance, if the given $h$ and $e$ fall in region 1 of Fig. 3, i.e., $h \geqslant 4$ and $e>e_{1}$, then there is only one equilibrium configuration $P_{0}$, as stated in the rule associated with Eq. (19). For the parameter ranges in Fig. 3 there are six regions. The equilibrium configurations in 


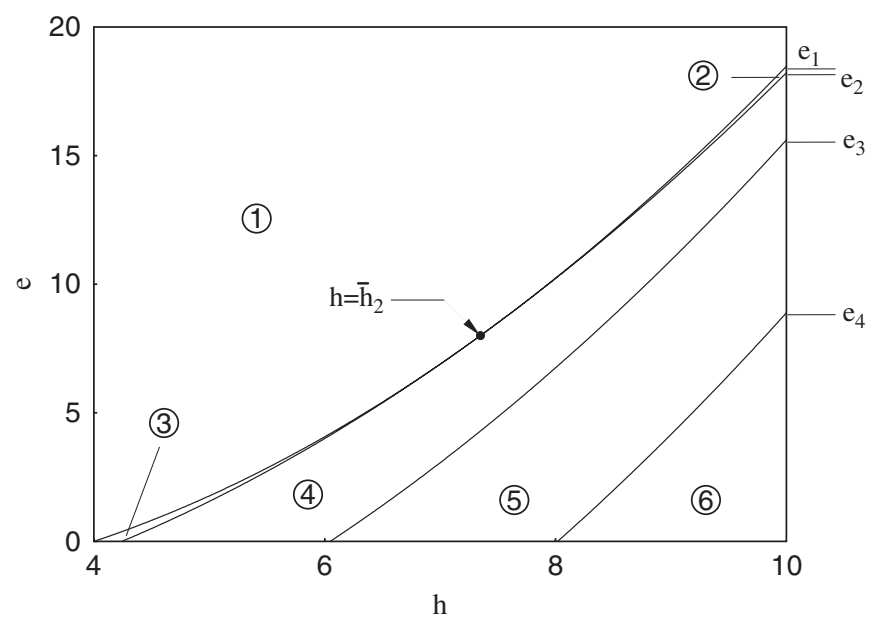

Fig. 3. $e_{j}$-curves on the $h-e$ plane.

Table 1

Equilibrium configurations and stability of various regions in Fig. 3

\begin{tabular}{lll}
\hline Region & Equilibrium configuration & Stability of $P_{1}^{-}$ \\
\hline 1 & $P_{0}$ & \\
2 & $P_{0}, P_{1}^{ \pm}$ & Unstable \\
3 & $P_{0}, P_{1}^{ \pm}$ & Stable \\
4 & $P_{0}, P_{1}^{ \pm}, P_{12}^{ \pm}$ & Stable \\
5 & $P_{0}, P_{1 \pm}^{ \pm}, P_{12}^{ \pm}, P_{13}^{ \pm}$ & Stable \\
6 & $P_{0}, P_{1}^{ \pm}, P_{12}^{ \pm}, P_{13}^{ \pm}, P_{14}^{ \pm}$ & Stable \\
\hline
\end{tabular}

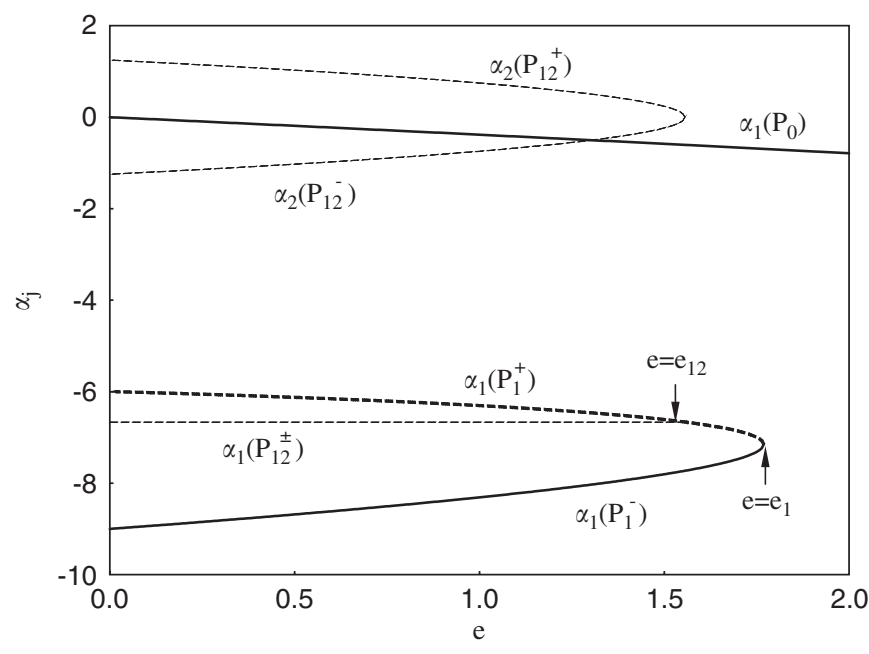

Fig. 4. Equilibrium configurations for $h=5$.

each region are listed in Table 1 . In the table we also note that the position in region 2 is unstable, while $P_{1}^{-}$in other regions are stable.

Fig. 4 shows the $\alpha_{j}-e$ curves for an arch with $h=5$, which is smaller than $3 \sqrt{6}$. For this arch $e_{1}=1.77$, and $e_{2}=1.56$. The thick and the thin lines represent the one-mode and two-mode solutions, respectively. The solid and dashed lines signify that the solutions are stable and unstable, respectively. We notice

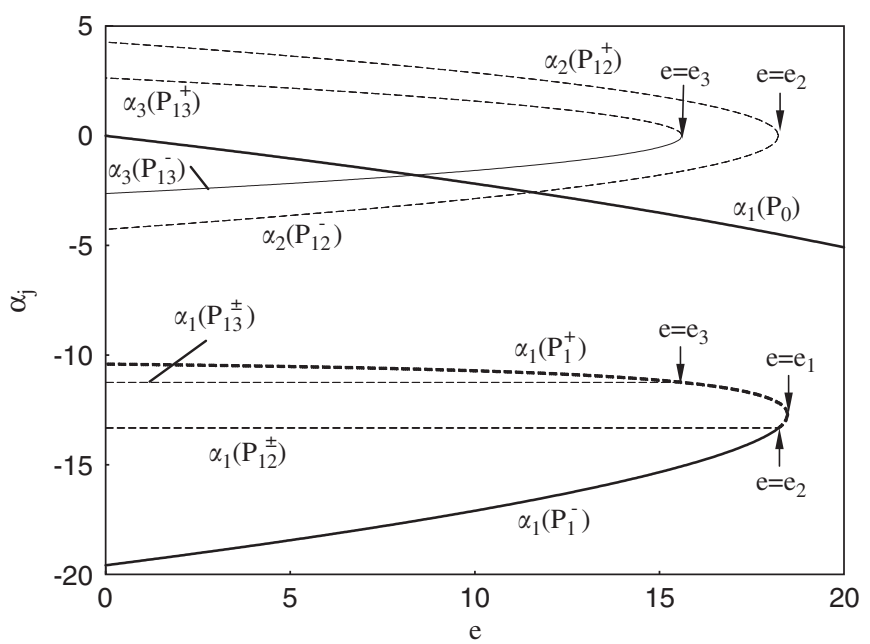

Fig. 5. Equilibrium configurations for $h=10$.

that at $e=0, \alpha_{1}\left(P_{12}^{ \pm}\right)$lies between $\alpha_{1}\left(P_{1}^{+}\right)$and $\alpha_{1}\left(P_{1}^{-}\right)$. As $e$ increases, $\alpha_{1}\left(P_{1}^{+}\right)$decreases and $\alpha_{1}\left(P_{1}^{-}\right)$increases, and finally they merge at $e=e_{1} \cdot \alpha_{1}\left(P_{12}^{ \pm}\right)$-curve remains horizontal because it is independent of $e$. We also observe that $\alpha_{1}\left(P_{1}^{+}\right)$ meets $\alpha_{1}\left(P_{12}^{ \pm}\right)$at $e=e_{2}$. The coordinates $\alpha_{2}$ of the two-mode solutions $P_{12}^{+}$and $P_{12}^{-}$also merge at $e=e_{2}$. For $e>e_{2}$ the two-mode solutions $P_{12}^{+}$and $P_{12}^{-}$cease to exist. Furthermore, configuration $P_{1}^{-}$remains stable as $e$ changes from 0 to $e_{1}$.

We now proceed to another case in Fig. 5 when $h=10$, which is greater than $3 \sqrt{6}$. For this $\operatorname{arch} e_{1}=18.47, e_{2}=18.22$, and $e_{3}=$ 15.61. We notice that for this case $\alpha_{1}\left(P_{1}^{-}\right)$, instead of $\alpha_{1}\left(P_{1}^{+}\right)$ as in Fig. 4, meets $\alpha_{1}\left(P_{12}^{ \pm}\right)$at $e=e_{2}$. The coordinates $\alpha_{2}$ of the two-mode solutions $P_{12}^{+}$and $P_{12}^{-}$also merge at $e=e_{2}$. In addition, $\alpha_{1}\left(P_{1}^{+}\right)$meets $\alpha_{1}\left(P_{13}^{ \pm}\right)$at $e=e_{3}$. The coordinates $\alpha_{3}$ of the two-mode solutions $P_{13}^{+}$and $P_{13}^{-}$also merge at $e=e_{3}$. For $e>e_{3}$ the two-mode solutions $P_{13}^{+}$and $P_{13}^{-}$cease to exist. Furthermore, configuration $P_{1}^{-}$remains stable as $e$ changes from 0 to $e_{2}$, instead of $e_{1}$ as in Fig. 4. It is noted from Figs. 4 and 5 that $\alpha_{1}\left(P_{0}\right)$ decreases monotonically as $e$ increases. Therefore, if the arch is stretched quasi-statically, the only possible deformation configuration of the arch is $P_{0}$. However, if the arch is stretched dynamically, it is possible for the arch to jump to the configuration $P_{1}^{-}$and stay there, as will be demonstrated numerically later.

\section{Energy barrier}

In the case when the end speed is not negligible dynamic snap-through may occur. If there exists only one stable equilibrium configuration when the motion of the arch end stops, this configuration must be $P_{0}$. In such a case the arch will return to this stable position after the damping effects kick in because it has nowhere else to settle. Therefore, for dynamic snap-through to occur there must exist at least two stable equilibrium configurations when the motion of the arch end stops. While it is in general difficult to determine the necessary and sufficient condition for dynamic snap-through to occur, we can establish 
the sufficient conditions against dynamic snap-through in terms of strain energy $U$ of the equilibrium configurations and the total energy $H$ gained by the arch at the instant when the arch end stop.

The basic idea is that if the total energy gained by the arch during the prescribed end motion is smaller than the minimum energy barrier lying between the nearest stable equilibrium position and another stable position farther away, then the arch has no chance to snap dynamically. The energy barrier can be proved to be the strain energy of either the unstable configuration $P_{1}^{+}$or $P_{12}^{ \pm}$, depending on the parameters $h$ and $e$. In summary, we have two cases. (1) First of all for a lower arch with $4<h \leqslant 3 \sqrt{6}$, the energy barrier is the strain energy $U\left(P_{12}^{ \pm}\right)$ when $e<e_{2}$. On the other hand when $e_{2} \leqslant e<e_{1}$, the energy barrier is $U\left(P_{1}^{+}\right)$. For $e>e_{1}$, there exists only one stable equilibrium position. (2) Secondly for a higher arch with $h>3 \sqrt{6}$, the energy barrier is $U\left(P_{12}^{ \pm}\right)$when $e<e_{2}$. For $e>e_{2}$, there exists only one stable equilibrium position.

After establishing the energy barrier, the remaining task is to determine the total energy $H$ gained by the arch at the instant $\tau_{s}$ when the arch end stops. However, to determine $H\left(\tau_{s}\right)$ we need to integrate the equations of motion directly, which requires tedious numerical work each time a new speed $c$ or distance $e$ is specified. Instead of calculating $H\left(\tau_{s}\right)$, we propose to estimate the upper bound of total energy gained by the arch during the end motion, as demonstrated in the next section.

\section{Upper bound of total energy}

We first assume that the initial conditions are

$\alpha_{n}(0)=\dot{\alpha}_{n}(0)=0, \quad n=1,2,3, \ldots$.

Because the equations of motion (10) for $n \neq 1$ have no explicit forcing term we have $\alpha_{n}(\tau) \equiv 0$ for $n \neq 1$. As a consequence, $G$ in Eq. (11) can be simplified to

$G=\frac{\alpha_{1}^{2}}{4}+\frac{h \alpha_{1}}{2}$.

By using Eq. (12) one can transform $\alpha(\tau)$ to $\alpha(e)$ and rewrite Eq. (9) as

$c^{2} \alpha_{1}^{\prime \prime}(e)=-\alpha_{1}(e)-(G+e)\left(h+\alpha_{1}(e)\right)$.

The superposed primes represent differentiation with respect to $e$. The initial conditions for $\alpha_{1}(e)$ are

$\alpha_{1}(0)=\alpha_{1}^{\prime}(0)=0$.

The total energy $H$ in Eq. (33) can be rewritten as

$H(e)=c^{2} \alpha_{1}^{\prime 2}(e)+\alpha_{1}^{2}(e)+2(G+e)^{2}$.

In the case when $c \rightarrow \infty$, we have from Eq. (57) that $\alpha_{1}^{\prime \prime}(e) \rightarrow 0$. From initial conditions (58), we also obtain $\alpha_{1}(e) \rightarrow 0$ and $\alpha_{1}^{\prime}(e) \rightarrow 0$. As a consequence, $G \rightarrow 0$ from Eq. (56). The corresponding total energy is denoted by $H_{\infty}$, where

$H_{\infty} \rightarrow 2 e^{2}$
The physical meaning of Eq. (60) is that when the arch is stretched with infinite speed, the arch has no time to move laterally, and the work done by the external force is stored in the form of strain energy due to axial elongation. We have no intention to argue whether infinite speed is physically feasible or not. Instead, we assert in the following theorem that $H_{\infty}=2 e^{2}$ is an upper bound of total energy gained by the arch during any prescribed end motion with finite speed.

Theorem 6.1. The total energy gained by the arch during prescribed end motion with finite speed is less than $2 e^{2}$.

Proof. From Eqs. (57) and (58) one can derive

$\alpha_{1}^{\prime \prime \prime}(0)=-\frac{h}{c^{2}}$.

In other words, $\alpha_{1}(e)$ may be expanded as

$\alpha_{1}(e)=-\frac{h}{6 c^{2}} e^{3}+$ high order terms.

Therefore, $\alpha_{1}<0$ for small enough $e$. From Eqs. (59) and (60) one can derive at $e=0$ :

$\begin{aligned} H_{\infty}-H & =\frac{d\left(H_{\infty}-H\right)}{\mathrm{d} e}=\frac{d^{2}\left(H_{\infty}-H\right)}{\mathrm{d} e^{2}}=\frac{d^{3}\left(H_{\infty}-H\right)}{\mathrm{d} e^{3}} \\ & =0\end{aligned}$

and

$\frac{d^{4}\left(H_{\infty}-H\right)}{\mathrm{d} e^{4}}=\frac{2 h^{2}}{c^{2}}$.

Therefore, $H_{\infty}-H$ may be expanded as

$H_{\infty}-H=\frac{h^{2}}{12 c^{2}} e^{4}+$ high order terms.

Consequently, $H_{\infty}-H>0$ for small enough $e$. If there exists an $\tilde{e}$ such that $H_{\infty}-H(\tilde{e})=0$ and $H_{\infty}-H(e)>0$ for any $e<\tilde{e}$, then from the mean value theorem and together with Eq. (63) there must exist an $\hat{e}$ with $0<\hat{e}<\tilde{e}$ such that

$\frac{d\left(H_{\infty}-H(\hat{e})\right)}{\mathrm{d} e}=0$.

From Eqs. (59) and (60), one can derive

$\frac{d\left(H_{\infty}-H\right)}{\mathrm{d} e}=-\alpha_{1}^{2}-2 h \alpha_{1}$

Therefore, for Eq. (66) to hold, either $\alpha_{1}(\hat{e})=0$ or $\alpha_{1}(\hat{e})=-2 h$. For the case of $\alpha_{1}(\hat{e})=0$, one has

$H(\hat{e})=c^{2} \alpha_{1}^{\prime 2}(\hat{e})+2 \hat{e}^{2} \geqslant H_{\infty}$,

which contradicts the original assumption. On the other hand, for the case of $\alpha_{1}(\hat{e})=-2 h$, one has

$H(\hat{e})=c^{2} \alpha_{1}^{\prime 2}(\hat{e})+\alpha_{1}^{2}(\hat{e})+2 \hat{e}^{2} \geqslant H_{\infty}$,

which contradicts the original assumption again. Therefore, $\hat{e}$ does not exist, and neither does $\tilde{e}$. Consequently, $H_{\infty}-H>0$ for all $e \neq 0$. 


\section{Snap-through criterion}

After establishing the upper bound of the total energy, we can state the sufficient conditions against dynamic snap-through in a more conservative way as follows:

Case (1): For a lower arch with $4<h \leqslant 3 \sqrt{6}$ : if $e_{2} \leqslant e<e_{1}$, then the sufficient condition against snap-through is $2 e^{2}<$ $U\left(P_{1}^{+}\right)$. If $e<e_{2}$, then the sufficient condition against snapthrough is $2 e^{2}<U\left(P_{12}^{ \pm}\right)$.

Case (2): For a higher arch with $h>3 \sqrt{6}$ : if $e<e_{2}$ then the sufficient condition against snap-through is $2 e^{2}<U\left(P_{12}^{ \pm}\right)$.

It can be further shown that the sufficient condition in case (1) is always satisfied, as stated in the next theorem.

Theorem 7.1. No snap-through will occur if $4<h \leqslant 3 \sqrt{6}$.

Proof. First of all it can be shown that

$\frac{\partial\left[U\left(P_{1}^{+}\right)-H_{\infty}\right]}{\partial e}=-\frac{4 \alpha_{1}\left(P_{1}^{+}\right)}{\alpha_{1}\left(P_{1}^{+}\right)+h}-4 e<0$.

In the case when $e=e_{1}$, it can be shown that

$$
\begin{aligned}
U\left(P_{1}^{+}\right)-H_{\infty}= & -\frac{h^{4}}{8}+3 \times 2^{-4 / 3} h^{8 / 3} \\
& +2 h^{2}-6 \times 4^{-4 / 3} h^{4 / 3} \geqslant 0 .
\end{aligned}
$$

From Eqs. (70) and (71) we know that $U\left(P_{1}^{+}\right)-H_{\infty}>0$ when $e_{2}<e<e_{1}$. We next show that $\alpha_{1}$ can never surpass $\alpha_{1}\left(P_{1}^{+}\right)$. We first assume that there exists an $\hat{e}$ such that $\alpha_{1}(\hat{e})=\alpha_{1}\left(P_{1}^{+}\right)$. If this does occur, then

$H(\hat{e})=c^{2} \alpha_{1}^{\prime 2}(\hat{e})+U\left(P_{1}^{+}\right)>U\left(P_{1}^{+}\right)$,

which contradicts the previous conclusion that $H(\hat{e})$ can never surpass $U\left(P_{1}^{+}\right)$. Therefore, $\alpha_{1}>\alpha_{1}\left(P_{1}^{+}\right)$always. This completes the proof that no snap-through will occur when $e_{2} \leqslant e<e_{1}$. The proof for the case $e<e_{2}$ is similar.

For case (2) when $h>3 \sqrt{6}$ and $e<e_{2}$, the critical energy barrier is $U\left(P_{12}^{ \pm}\right)$. It can be found that there exist an $e_{\mathrm{cr}}$, such that

$H_{\infty}\left(e_{\mathrm{cr}}\right)=U\left(P_{12}^{ \pm}\right)$,

where $e_{\text {cr }}$ can be calculated as

$e_{\mathrm{cr}}=\sqrt{\frac{8}{3}} h-4$.

Similar to the proof in Theorem 7.1, one can prove the following theorem.

Theorem 7.2. No snap-through will occur if $h>3 \sqrt{6}$ and $e<e_{\mathrm{cr}}$.

From Theorems 7.1 and 7.2 we conclude that dynamic snapthrough might occur only when $h>3 \sqrt{6}$ and $e_{\mathrm{cr}}<e<e_{2}$, or more explicitly, $\sqrt{\frac{8}{3}} h-4<e<\frac{2}{9} h^{2}-4$. Region I in Fig. 6

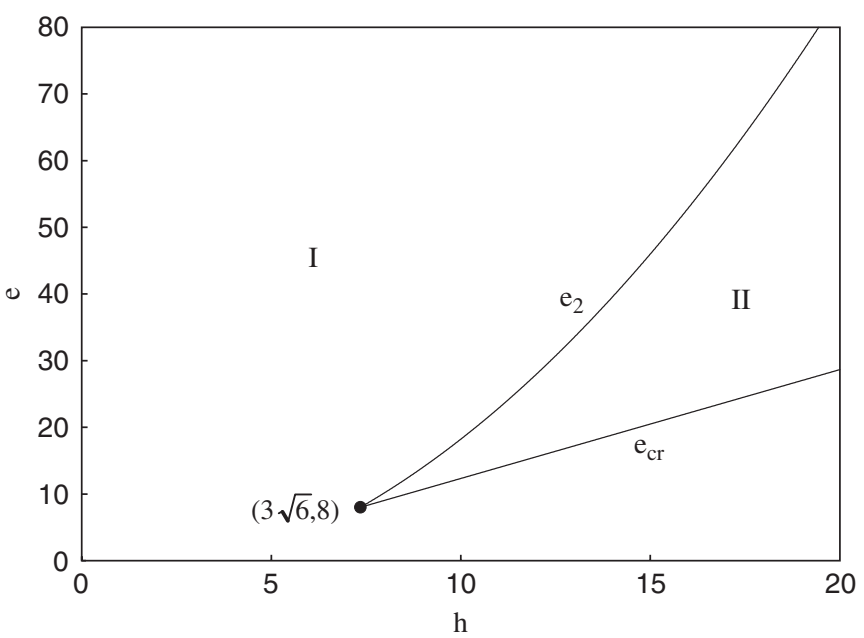

Fig. 6. Boundary for sufficient conditions against dynamic snap-through under prescribed end motion. For those parameters in region I no snap-through is possible. For those parameters in region II dynamic snap-through may or may not occur, depending on the stretching speed.

represents the region within which no snap-through is possible, no matter how fast the speed $c$ is. On the other hand, region II represents the region within which snap-through might occur. The boundary curves $e=e_{2}$ and $e=e_{\mathrm{cr}}$ intersect at the point $(h, e)=(3 \sqrt{6}, 8)$.

Fig. 7 shows the total energy as a function of $e$ for various speeds. The $H_{\infty}$ curve calculated from Eq. (60) represents the upper bound of total energy for any finite speed. In Fig. 7(a) for $h=5<3 \sqrt{6}$, the energy barriers are $U\left(P_{12}^{ \pm}\right)$and $U\left(P_{1}^{+}\right)$ when $e$ is in the ranges $0<e<e_{2}=1.56$ and $e_{2}<e<e_{1}=1.77$, respectively. The readers are reminded that configurations $P_{12}^{+}$ and $P_{12}^{-}$do not exist for $e$ in the range $e_{2}<e<e_{1}$. The total energy upper bound $H_{\infty}$ can never surpass the energy barrier in time when $e$ changes from 0 to 2 . The energy history curve for $c=5$ is also shown. The energy history curves for $c>5$ are squeezed between $H_{\infty}$ and the curve for $c=5$.

In Fig. 7(b) for $h=10>3 \sqrt{6}, H_{\infty}$ curve meets $U\left(P_{12}^{ \pm}\right)$ curve at $e_{\mathrm{cr}}=12.33$, which is smaller than $e_{2}=18.22$. $U\left(P_{12}^{ \pm}\right)$ is the energy barrier in this case. The energy history curves for four speeds $c=15,31.9,50$, and 100 are shown to approach $H_{\infty}$ as $c$ increases. Among them $c=31.9$ is defined as a critical speed because it is the lowest stretching speed for the arch to gain enough total energy to surpass the energy barrier. The concept of critical speed will be explained in more detail in the next section. Fig. 7(c) shows the special case when $h=3 \sqrt{6}$, at which $H_{\infty}$ curve meets $U\left(P_{1}^{+}\right)$and $U\left(P_{12}^{+}\right)$curves at $e=$ $e_{\mathrm{cr}}=e_{1}=e_{2}=8$.

Fig. 8 shows the deformation history for the arch with $h=10$, as in Fig. 7(b). In calculating the response we modify Eq. (9) by adding a damping parameter $\mu$,

$\ddot{\alpha}_{1}=-\mu \dot{\alpha}_{1}-\alpha_{1}-(G+e)\left(h+\alpha_{1}\right)$.

The speed and damping are set to be $c=1000$, and $\mu=0.001$. Fig. 8(a) shows the case when $e=10<e_{\mathrm{cr}}$. For this case the time when the moving end stops is $\tau_{s}=0.01$. Fig. 8 (b) shows the 


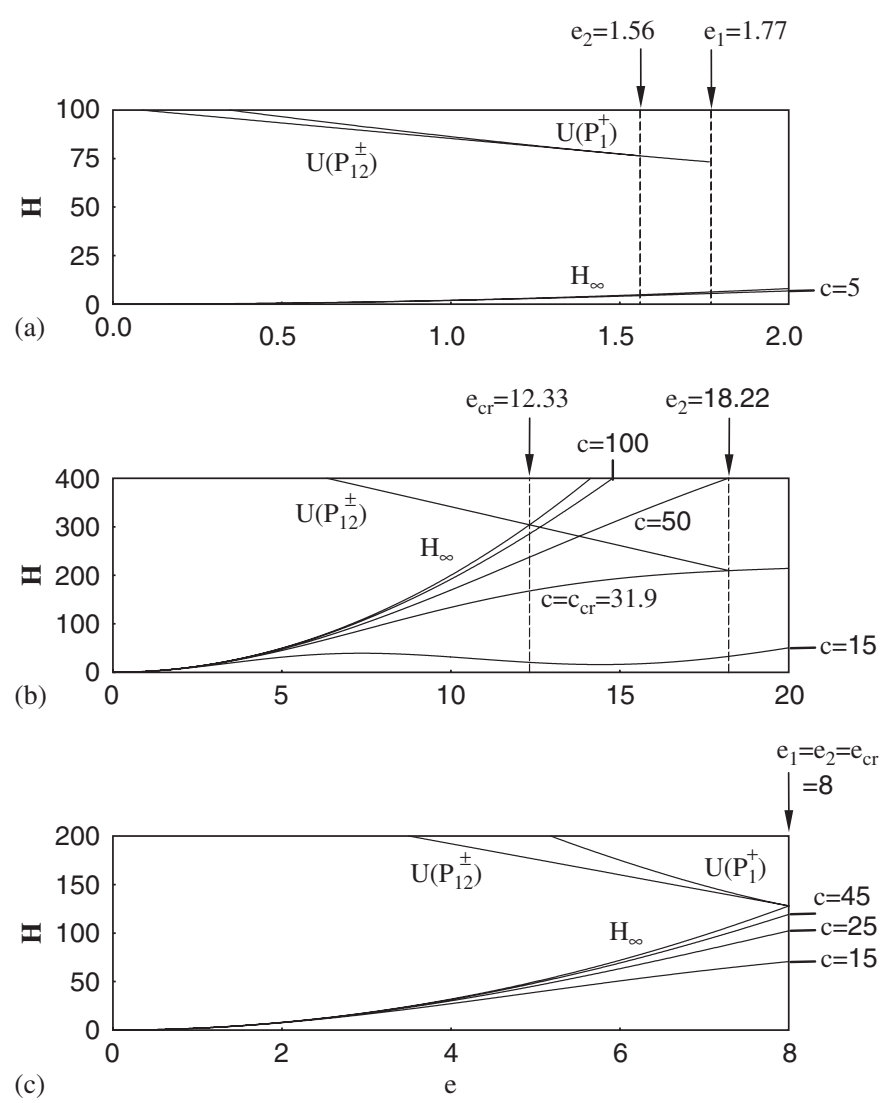

Fig. 7. Total energy history for various stretching speeds: (a) $h=5$; (b) $h=10$; (c) $h=3 \sqrt{6}$.
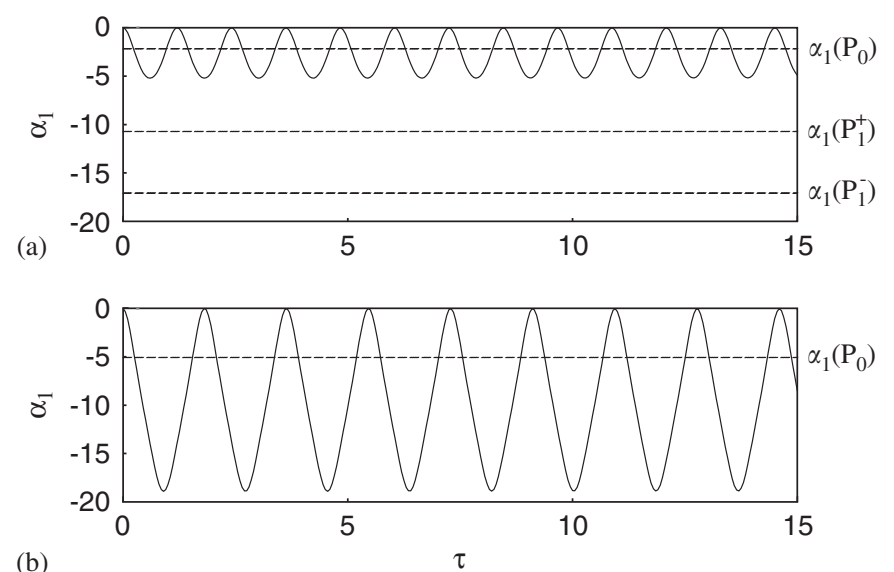

(b)

Fig. 8. Response history for $h=10, c=1000$, and $\mu=0.001$ : (a) $e=10$, (b) $e=20$. The $(h, e)$ parameters in both cases fall in range I of Fig. 6.

case when $e=20>e_{2}$. For this case $\tau_{s}=0.02$. The equilibrium solutions $\alpha_{1}$ are also plotted as dashed lines for reference. In both cases the arch settles to configuration $P_{0}$ as expected.

Figs. 9(a)-(c) show the deformation history for an arch with $h=10$, and $e=15$, which falls in region II of Fig. 6 . The speed $c$ is set to be 50 . For these cases $\tau_{s}=0.3$, as signified by the black dots on the response curves. The damping $\mu$ used in Figs. 9(a)-(c) are $0.005,0.01$, and 0.05 , respectively. For the

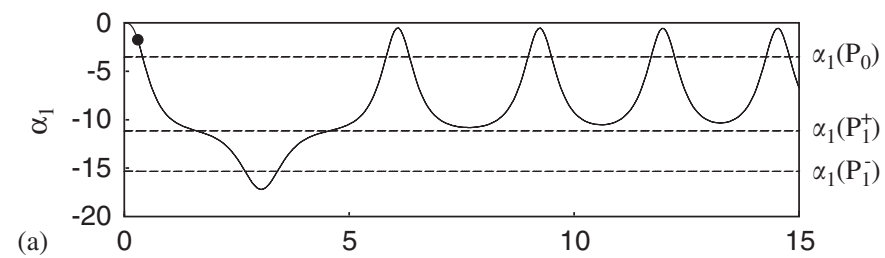

(b)
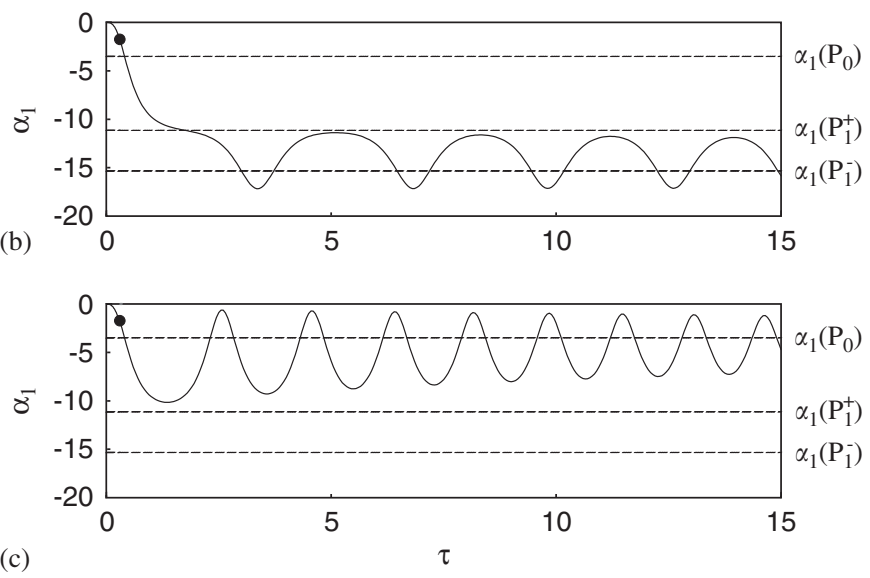

Fig. 9. Response history of an arch with $c=50, h=10, e=15$, which falls in range II of Fig. 6: (a) $\mu=0.005$; (b) $\mu=0.01$; (c) $\mu=0.05$.

small damping case in Fig. 9(a) the energy gained by the arch is large enough to surpass $U\left(P_{12}^{ \pm}\right)$and $\alpha_{1}$ can reach $\alpha_{1}\left(P_{1}^{-}\right)$. However, due to small damping, the arch is snapped back and finally settles to the configuration $P_{0}$. For the medium damping case in Fig. 9(b), the arch not only gains enough energy to surpass $U\left(P_{12}^{ \pm}\right)$and $\alpha_{1}$ can reach $\alpha_{1}\left(P_{1}^{-}\right)$, the damping also prevents it from snapping back to $P_{0}$. The arch settles to $P_{1}^{-}$ eventually. This phenomenon is called dynamic snap-through under prescribed end motion. In Fig. 9(c) the damping is so large that it prevents the arch from surpassing the energy barrier $U\left(P_{12}^{ \pm}\right)$, and the arch has no choice but to settle to $P_{0}$.

\section{Critical stretching speed}

In Fig. 9 we have shown that even if the parameters $e$ and $h$ fall in region II of Fig. 6, snap-through may or may not occur depending on the damping parameter. Another important factor in determining whether snap-through will occur is the stretching speed. Apparently, for very small stretching speed no snap-through is possible because the arch will remain in $P_{0}$. As $c$ increases, for instance up to 50 as shown in Fig. 9, snapthrough might occur. It is therefore possible to define a critical stretching speed $c_{\mathrm{cr}}$ below which no snap-through is possible even if $e$ and $h$ fall in region II of Fig. 6.

Fig. 10 shows the critical stretching speed as a function of $h$. For each $h$, we integrate Eq. (57) for various stretching speeds $c$, and calculate the total energy from Eq. (59) for all $e$ in the range $e_{\mathrm{cr}}<e<e_{2}$. The minimum $c$ which renders $H(e)=U\left(P_{12}^{ \pm}\right)$is defined as the critical stretching speed. Numerical calculation shows that the minimum critical speed is 25.9 which occurs at $h=8.4$. As $h$ approaches $3 \sqrt{6}$, the critical speed approaches infinity. Therefore, as long as the stretching speed is below 


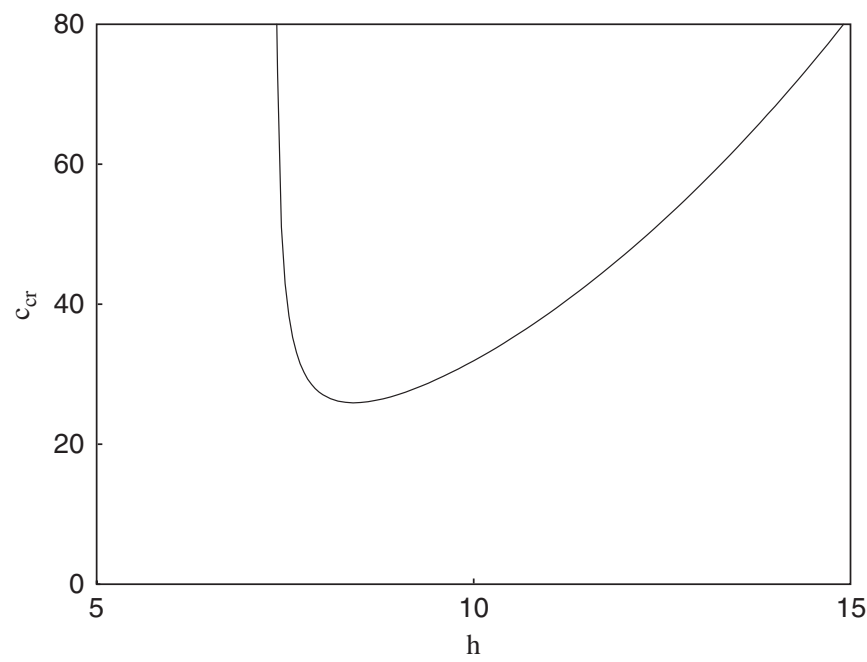

Fig. 10. Critical stretching speed $c_{\mathrm{cr}}$ as a function of $h$. No snap-through is possible when the stretching speed $c$ is smaller than $c_{\mathrm{cr}}$.

25.9, no snap-through is possible for all possible combinations of $e$ and $h$.

\section{Conclusions}

In this paper we consider a shallow arch with rise parameter $h$, free of lateral loading, but subject to prescribed end motion $e$ with constant speed $c$. Some conclusions can be summarized in the following:

(1) For $h<4$, or $h \geqslant 4$ but $e>e_{1}$, there is only a one-mode equilibrium configuration $P_{0}$. For $h>4$ and $e<e_{1}$, there are three one-mode equilibrium configurations $P_{0}, P_{1}^{+}$, and $P_{1}^{-}$. Two-mode solutions $P_{1 j}^{+}$and $P_{1 j}^{-}$exist only when $e<e_{j}$.

(2) There are at most two stable equilibrium configurations for any given $e$ and $h$. One of them is $P_{0}$, which is always stable. The other is $P_{1}^{-}$, which is stable only in certain range of $e$ and $h$.

(3) The total energy gained by the arch has an upper bound $2 e^{2}$, which corresponds to the case when $c$ approaches infinity.

(4) The only possible situation when dynamic snap-through may occur is $h>3 \sqrt{6}$ and $e_{\mathrm{cr}}<e<e_{2}$, where $e_{\mathrm{cr}}=\sqrt{\frac{8}{3}} h-$ 4 and $e_{2}=\frac{2}{9} h^{2}-4$. In this case, to prevent dynamic snapthrough to occur the end speed $c$ must not exceed a critical speed $c_{\mathrm{cr}}$. The minimum critical stretching speed is found to be 25.9 for all possible combinations of $e$ and $h$.

\section{References}

[1] S.P. Timoshenko, Buckling of flat curved bars and slightly curved plates, ASME J. Appl. Mech. 2 (1935) 17-20.

[2] Y.C. Fung, A. Kaplan, Buckling of low arches or curved beams of small curvature, NACA Technical Note 2840, 1952.
[3] A. Gjelsvik, S.R. Bonder, The energy criterion and snap buckling of arches, ASCE J. Eng. Mech. Div. 88 (1962) 87-134.

[4] E.T. Onat, L.S. Shu, Finite deformation of a rigid perfectly plastic arch, ASME J. Appl. Mech. 29 (1962) 549-553.

[5] V. Franciosi, G. Augusti, R. Sparacio, Collapse of arches under repeated loading, ASCE J. Struct. Div. 90 (1964) 165-201.

[6] H.L. Schreyer, E.F. Masur, Buckling of shallow arches, ASCE J. Eng. Mech. Div. 92 (1966) 1-19.

[7] H.N. Lee, L.M. Murphy, Inelastic buckling of shallow arches, ASCE J. Eng. Mech. Div. 94 (1968) 225-239.

[8] E.F. Masur, D.L.C. Lo, The shallow arch-general buckling, post buckling, and imperfection analysis, J. Struct. Mech. 1 (1972) 91-112.

[9] G.J. Simitses, Snapping of low pinned arches on an elastic foundation, ASME J. Appl. Mech. 40 (1973) 741-744.

[10] J. Roorda, Stability of structures with small imperfections, ASCE J. Eng. Mech. Div. 91 (1965) 87-106.

[11] N.J. Hoff, V.G. Bruce, Dynamic analysis of the buckling of laterally loaded flat arches, J. Math. Phys. 32 (1954) 276-288.

[12] J.S. Humphreys, On dynamic snap buckling of shallow arches, AIAA J. 4 (1966) 878-886.

[13] M.H. Lock, The snapping of a shallow sinusoidal arch under a step pressure load, AIAA J. 4 (1966) 1249-1256.

[14] C.S. Hsu, The effects of various parameters on the dynamic stability of a shallow arch, ASME J. Appl. Mech. 34 (1967) 349-358.

[15] C.S. Hsu, Stability of shallow arches against snap-through under timewise step loads, ASME J. Appl. Mech. 35 (1968) 31-39.

[16] C.S. Hsu, C.T. Kuo, S.S. Lee, On the final states of shallow arches on elastic foundations subjected to dynamical loads, ASME J. Appl. Mech. 35 (1968) 713-723.

[17] N.N. Huang, W. Nachbar, Dynamic snap-through of imperfect viscoelastic shallow arches, ASME J. Appl. Mech. 35 (1968) 289-296.

[18] S.T. Ariaratnam, T.S. Sankar, Dynamic snap-through of shallow arches under stochastic loads, AIAA J. 6 (1968) 798-802.

[19] R.E. Fulton, F.W. Barton, Dynamic buckling of shallow arches, ASCE J. Eng. Mech. Div. 97 (1971) 865-877.

[20] V. Sundararajan, D.S. Kumani, Dynamic snap-buckling of shallow arches under inclined loads, AIAA J. 10 (1972) 1090-1091.

[21] D.L.C. Lo, E.F. Masur, Dynamic buckling of shallow arches, ASCE J. Eng. Mech. Div. 102 (1976) 901-917.

[22] E.R. Johnson, I.K. Mclvor, The effect of spatial distribution on dynamic snap-through, ASME J. Appl. Mech. 45 (1978) 612-618.

[23] E.R. Johnson, The effect of damping on dynamic snap-through, ASME J. Appl. Mech. 47 (1980) 601-606.

[24] K.Y. Huang, R.H. Plaut, Snap-through of a shallow arch under pulsating load, in: F.H. Schroeder (Ed.), Stability in the Mechanics of Continua, Springer, Berlin, 1982, pp. 215-223.

[25] W.E. Gregory Jr., R.H. Plaut, Dynamic stability boundaries for shallow arches, ASCE J. Eng. Mech. Div. 108 (1982) 1036-1050.

[26] M.T. Donaldson, R.H. Plaut, Dynamic stability boundaries for a sinusoidal arch under pulse loads, AIAA J. 21 (1983) 469-471.

[27] P. Patricio, M. Adda-Bedia, M.B. Amar, An elastica problem: instabilities of an elastic arch, Physica D 124 (1998) 285-295.

[28] J.-X. Xu, H. Huang, P.-Z. Zhang, J.-Q. Zhou, Dynamic stability of shallow arch with elastic supports-application in the dynamic stability analysis of inner winding of transformer during short circuit, Int. J. Non-Linear Mech. 37 (2002) 909-920. 